\section{Disease-modifying drugs for multiple sclerosis and subsequent health service use}

\author{
Huah Shin Ng iD, Feng Zhu, Elaine Kingwell, Yinshan Zhao, Shenzhen Yao, \\ Okechukwu Ekuma, Lawrence W Svenson, Charity Evans, John D Fisk, \\ Ruth Ann Marrie ${ }^{\text {iD }}$ and Helen Tremlett ${ }^{\text {iD }}$
}

\section{Abstract}

Objective: We assessed the relationship between the multiple sclerosis (MS) disease-modifying drugs (DMDs) and healthcare use.

Methods: Persons with MS (aged $\geqslant 18$ years) were identified using linked population-based health administrative data in four Canadian provinces and were followed from the most recent of their first MS/ demyelinating event or 1 January 1996 until the earliest of death, emigration, or study end (31 December 2017 or 31 March 2018). Prescription records captured DMD exposure, examined as any DMD, then by generation (first-generation (the injectables) or second-generation (orals/infusions)) and individual DMD. The associations with subsequent all-cause hospitalizations and physician visits were examined using proportional means model and negative binomial regression.

Results: Of 35,894 MS cases (72\% female), mean follow-up was 12.0 years, with person-years of DMD exposure for any, or any first- or second-generation DMD being 63,290, 54,605 and 8685, respectively. Any DMD or any first-generation DMD exposure (versus non-exposure) was associated with a $24 \%$ lower hazard of hospitalization (adjusted hazard ratio, aHR: 0.76 ; 95\% confidence intervals (CIs): $0.71-$ 0.82), rising to $29 \%$ for the second-generation DMDs (aHR: 0.71 ; 95\% CI: 0.58-0.88). This ranged from $18 \%$ for teriflunomide (aHR: 0.82 ; 95\% CI: $0.67-1.00$ ) to 44\% for fingolimod (aHR: $0.56 ; 95 \%$ CI: $0.36-0.87)$. In contrast, DMD exposure was generally not associated with substantial differences in physician visits.

Conclusion: Findings provide real-world evidence of a beneficial relationship between DMD exposure and hospitalizations.

Keywords: Disease-modifying drugs, health services, hospitalization, multiple sclerosis, physician services

Date received: 7 September 2021; revised: 26 October 2021; accepted: 11 November 2021

\section{Introduction}

Disease-modifying drug (DMD) efficacy is typically established after short clinical trials in highly selected multiple sclerosis (MS) patients. ${ }^{1}$ However, in clinical practice, DMDs are used in the wider MS population. A recent study showed that nearly $20 \%$ of people with MS had some comorbidity and approximately one in six were aged $\geqslant 50$ years when first receiving a DMD. ${ }^{2}$ However, such individuals are typically excluded from clinical trials. ${ }^{2}$ Understanding the benefits of the DMDs in the broader MS populations treated in the "real-world" setting is needed for therapeutic-related decision-making. Health service use, particularly hospitalizations, represents a major burden for society, patients, and families. Population-based longitudinal studies of health care use are needed to assess the benefits of the DMDs and facilitate healthcare planning. ${ }^{3}$ Prior studies have not been population-based, have been limited to private health insurance enrollees ${ }^{4,5}$ or were ecological in design and unable to assess DMD use at the individual patient level. 6,7

We investigated the relationship between DMD exposure and health service use in a MS population using individual patient-level linked administrative data collected over a 22-year period.
Multiple Sclerosis Journal

$1-14$

DOI: 10.1177

13524585211063403

(C) The Author(s), 2021

(c) (i)

Article reuse guidelines: sagepub.com/journals-

permissions

Correspondence to: H Tremlett

Division of Neurology, Department of Medicine, Faculty of Medicine, UBC Hospital, Room S126, 2211 Wesbrook Mall, Vancouver, BC V6T 2B5, Canada. helen.tremlett@ubc.ca

Huah Shin Ng Feng Zhu

Yinshan Zhao

Department of Medicine, Division of Neurology and the Djavad Mowafaghian Centre for Brain Health,

University of British

Columbia, Vancouver, BC,

Canada

Elaine Kingwell

Department of Medicine,

Division of Neurology and the Djavad Mowafaghian Centre for Brain Health,

University of British Columbia, Vancouver, BC, Canada/Research Department of Primary Care \& Population Health, University College London, London, UK

Shenzhen Yao

College of Pharmacy and Nutrition, University of Saskatchewan, Saskatoon, SK, Canada/Health Quality Council, Saskatoon, SK, Canada

Okechukwu Ekuma Department of Community Health Sciences, Rady Faculty of Health Sciences, University of Manitoba, Winnipeg, MB, Canada

Lawrence W Svenson Alberta Health, Edmonton, $\mathrm{AB}$, Canada/Division of Preventive Medicine \& School of Public Health, University of Alberta, Edmonton, AB, Canada/ Community Health Sciences, Cumming School of Medicine, University of Calgary, Calgary, AB, Canada

Charity Evans

College of Pharmacy and Nutrition, University of Saskatchewan, Saskatoon, SK, Canada 
John D Fisk

Nova Scotia Health

Authority and the

Departments of

Psychiatry, Psychology

and Neuroscience, and

Medicine, Dalhousie

University, Halifax, NS,

Canada

Ruth Ann Marrie

Departments of Internal

Medicine and Community

Health Sciences, Max Rady

College of Medicine, Rady

Faculty of Health Sciences,

University of Manitoba,

Winnipeg, MB, Canada

Helen Tremlett

Department of Medicine,

Division of Neurology an

the Djavad Mowafaghian

Centre for Brain Health,

University of British

Columbia, Vancouver,

BC, Canada/Division of

Neurology, Department

of Medicine, Faculty of

Medicine, UBC Hospital,

Vancouver, BC, Canada

\section{Methods}

\section{Data sources}

This was a multi-region, population-based observational study. Multiple administrative health databases covering four provinces (British Columbia, Saskatchewan, Manitoba, Nova Scotia), representing nearly $25 \%$ of Canada's population, ${ }^{8}$ were linked for each individual resident within each province. The administrative data included the following: physician and hospital data, ${ }^{9,10}$ capturing all physician visits and hospitalizations, coded using the International Classification of Diseases (ICD) system; the provincial health insurance registries, ${ }^{11}$ capturing demographic information (residency status, sex, birthdates, and residential postal codes, and for Manitoba/Saskatchewan, death dates); and vital statistics data, ${ }^{12}$ capturing death records in British Columbia/Nova Scotia. Prescription data provided all pharmacy dispensed prescriptions (including dates and number of days supplied/quantity dispensed; British Columbia, ${ }^{13}$ Manitoba, and Saskatchewan). In Nova Scotia, the Dalhousie MS Research Unit database provided DMD prescription start and stop dates.

\section{Study population}

We identified MS cases using a validated algorithm ( $\geqslant 3$ MS diagnostic codes (ICD-9/10 340/G35) or $\geqslant 1$ dispensation of a DMD). ${ }^{14}$ Once identified, cases were assigned an index date, defined as the most recent of the first MS or related demyelinating disease code captured in the physician/hospital data or first MS DMD prescription filled (Supplementary Tables 1-2); an individual's 18th birthday; or 1 January 1996 (British Columbia), 1 April 1996 (Manitoba), 1 January 1997 (Saskatchewan), or 1 January 1998 (Nova Scotia). These dates represent the first availability of prescription data (including the MS DMDs), and first full year of government financial coverage of the DMDs. One year of provincial residency preindex date was required; all identified persons with MS were followed from their index date until the earliest of death, emigration, or study end (31 December 2017 (British Columbia, Manitoba, Nova Scotia) and 31 March 2018 (Saskatchewan) based on the last availability date).

Cohort characteristics - sex, age, calendar-year, and socioeconomic status (estimated from neighborhoodlevel income via postal codes) ${ }^{15}$ - were described at the index date. Comorbidity in the year pre-index date was measured using the Charlson Comorbidity index (excluding hemiplegia and paraplegia to avoid misclassifying MS symptoms). ${ }^{16}$

\section{Exposure}

First- and second-generation DMD availability over the study period is shown in Supplementary Table 2, including the year of each DMD's approval for use in MS by Health Canada. Briefly, the first-generation DMDs included the injectables (i.e. beta-interferon and glatiramer acetate), and the second-generation included the infusions and orally administered DMDs. ${ }^{17}$ DMD exposure periods were determined based on the number of days supplied (British Columbia, Manitoba), or the quantity dispensed (Saskatchewan), or the DMD start/ stop dates (Nova Scotia). Discontinuation was defined as no further dispensations for that DMD (lasting $\geqslant 90$ consecutive days), plus a 30-day grace period (all provinces); that is, discontinuation date=last DMD dispensed date + days supplied +30 days. ${ }^{18}$ Except for alemtuzumab and ocrelizumab, whereby a person was considered exposed for a full 12- or 6-month postdispensation, respectively, and discontinued thereafter if no further dispensations (plus the 30-day grace period).

An individual's exposure status could change over time (i.e. time-dependent variable) and was assessed as periods of any exposure or no exposure, then by generation and individual DMD.

\section{Outcomes}

The primary and secondary outcomes were all-cause hospitalizations (events) and number of all physician service use (visits), respectively. Hospitalizations included day surgery, but not infusions (e.g. natalizumab or alemtuzumab). ${ }^{19}$ To avoid double counting, any new hospitalization occurring within 24 hours (e.g. due to hospital transfers) were considered one event. Evidence of $>1$ identical physician visitrelated ICD code on the same day was considered one visit.

Not counted as outcomes were pregnancy-related hospital or physician visit ICD codes (since DMD discontinuation is common during pregnancy) ${ }^{20}$ and neurologist specialty-specific physician visits (as their frequency is expected to be higher in persons using a DMD (versus not using), as per standard of care, ${ }^{20}$ and could result in the overestimation of healthcare use related to DMD prescribing).

\section{Statistical analyses}

Data were analyzed within each province using a common approach. Aggregated results were combined using random-effects meta-analyses. We assessed the association between DMD exposure and (1) all-cause hospitalizations using a recurrent events 
proportional means model with robust sandwich variance estimates and (2) physician visits using a negative binomial regression model fitted by generalized estimating equations with exchangeable working correlation matrix. Findings were expressed as hazard or rate ratios, with $95 \%$ confidence intervals (CIs). These approaches allow for multiple events analyses for each participant while accounting for dependence of events. For hospitalizations, because an individual cannot be at risk of a second hospitalization while already hospitalized, the hospital stay was excluded from the follow-up period. For physician visits, the number were counted yearly, or by periods of DMD exposure, if changes in DMD use occurred within a 1-year period; an offset ( $\log$ (days within each time period)) was specified to account for the variable length of periods. All models were adjusted for sex and socioeconomic status at the index date. Age, Charlson comorbidity score, and calendar-year were adjusted for as time-dependent covariates, updated on a yearly basis (the latter adjustment was made to account for any secular changes in healthcare delivery). The "DMDunexposed" period formed the reference category for the main analyses, although the pairwise comparisons between DMDs (by generation and individual DMD) were reported from the same models as an exploratory approach. Crude rates per 100 personyears of follow-up were described.

Complementary sex and physician specialty-specific analyses were performed. The latter was applied to the physician visits only, with crude rates by personyears of follow-up described for each DMD, and during periods of no exposure. In the largest province only, British Columbia, neurologist specialty was included in the count of all physician visits as a complementary approach. SAS V.9.4 and R V.4.0.2 were used for analyses.

\section{Results}

\section{Cohort characteristics}

A total of 35,894 MS cases were identified; most were female $(72 \%)$ and the mean age at index date was 44.6 (standard deviation $(\mathrm{SD})=13.6$ ) years (Table 1). Cohort characteristics, including by DMD exposure, are published in detail. ${ }^{2}$ Approximately $30 \%$ of our study population were exposed to a DMD during follow-up, and this estimate falls within the ranges reported in other population-based observational studies over a comparable time period. ${ }^{2}$ The total person-years of follow-up while exposed to any DMD was 63,290 (10,418 individuals); any first-generation
DMD was 54,605 (9204 individuals) and any secondgeneration DMD was 8685 (3668 individuals).

\section{Hospitalizations}

Exposure to any (versus no) DMD was associated with a 24\% lower hazard of hospitalization (adjusted hazard ratio, aHR: $0.76 ; 95 \%$ confidence interval (CI): 0.71-0.81). Similarly, for the firstgeneration DMDs, a $24 \%$ lower hazard was observed, with a $29 \%$ lower hazard for the second generation (Figure 1). Variation was seen across the individual DMDs, ranging from an 18\% lower hazard for teriflunomide, $20 \%$ for natalizumab, $22 \%$ for glatiramer acetate and alemtuzumab, 24\% for beta-interferon, to $36 \%$ for dimethyl fumarate and $44 \%$ for fingolimod. However, the HRs did not reach significance for teriflunomide and alemtuzumab (95\% CIs included one).

\section{Physician visits}

Overall, while exposure to any (versus no) DMD was associated with a lower rate of physician visits, this was modest and did not reach significance (adjusted rate ratio, aRR: 0.95; 95\% CI: 0.88-1.01). A similar pattern was observed for exposure to a first- or second-generation DMD and for most of the individual DMDs (Figure 2). Only glatiramer acetate was associated with a significantly lower rate of physician visits (by $9 \%$ ).

\section{Comparisons between DMDs}

When the DMDs were directly compared to each other, for hospitalizations, there was no significant difference between second- and first-generation DMDs (aHR: 0.87; 95\% CI: 0.66-1.17). When assessed by individual DMD, no differences were evident when the first-generation (beta-interferon or glatiramer acetate) were each compared to each of the other DMDs (Table 2). For the second-generation DMDs, natalizumab was associated with a $30 \%-49 \%$ higher hazard of hospitalization versus dimethyl fumarate and fingolimod.

For physician visits, while there was no significant difference for the second- versus first-generation DMDs (aRR: 1.01; 95\% CI: 0.94-1.08), rates differed by individual DMD (Table 3). For example, alemtuzumab was associated with a $19 \%-34 \%$ significantly higher rate of physician visits when compared to each of the other DMDs, while teriflunomide was associated with a $10 \%-12 \%$ higher rate when compared to glatiramer acetate, natalizumab, and fingolimod. 
Table 1. Characteristics of the multiple sclerosis study population, Canada (1996-2017/2018).

\begin{tabular}{|c|c|c|c|c|c|}
\hline Characteristics & $\begin{array}{l}\text { British } \\
\text { Columbia, } \\
n=19,360\end{array}$ & $\begin{array}{l}\text { Manitoba, } \\
n=5825\end{array}$ & $\begin{array}{l}\text { Nova Scotia, } \\
n=5352\end{array}$ & $\begin{array}{l}\text { Saskatchewan, } \\
n=5357\end{array}$ & $\begin{array}{l}\text { Overall cohort, } \\
n=35,894\end{array}$ \\
\hline \multicolumn{6}{|l|}{ Sex, $n(\%)$} \\
\hline Female & $13,940(72.0)$ & $4131(70.9)$ & $3989(74.5)$ & $3717(69.4)$ & $25,777(71.8)$ \\
\hline Male & $5420(28.0)$ & $1694(29.1)$ & $1363(25.5)$ & $1640(30.6)$ & $10,117(28.2)$ \\
\hline Age at index date in years, mean (SD) & $44.5(13.5)$ & $44.4(13.7)$ & $44.5(13.2)$ & $45.3(14.1)$ & $44.6(13.6)$ \\
\hline \multicolumn{6}{|l|}{ Socioeconomic status ${ }^{\mathrm{a}}, n(\%)$} \\
\hline 1 (lowest income quintile) & $3763(19.4)$ & $885(15.2)$ & $909(17.0)$ & $872(16.3)$ & $6429(17.9)$ \\
\hline 2 & $3695(19.1)$ & $1088(18.7)$ & $1030(19.2)$ & $1103(20.6)$ & $6916(19.3)$ \\
\hline 3 & $3931(20.3)$ & $1255(21.5)$ & $1043(19.5)$ & $1004(18.7)$ & $7233(20.2)$ \\
\hline 4 & $4094(21.1)$ & $1102(18.9)$ & $1034(19.3)$ & $1154(21.5)$ & $7384(20.6)$ \\
\hline 5 (highest income quintile) & $3779(19.5)$ & $1310(22.5)$ & $1014(18.9)$ & $988(18.4)$ & $7091(19.8)$ \\
\hline Unavailable & $98(0.5)$ & $185(3.2)$ & $322(6.0)$ & $236(4.4)$ & $841(2.3)$ \\
\hline \multicolumn{6}{|l|}{ Comorbidity score ${ }^{\mathrm{b}}, n(\%)$} \\
\hline 0 & $15,002(77.5)$ & $4525(77.7)$ & $4149(77.5)$ & $4297(80.2)$ & $27,973(77.9)$ \\
\hline 1 & $2965(15.3)$ & $963(16.5)$ & $850(15.9)$ & $796(14.9)$ & $5574(15.5)$ \\
\hline 2 & $903(4.7)$ & $235(4.0)$ & $215(4.0)$ & $174(3.2)$ & $1527(4.3)$ \\
\hline$\geqslant 3$ & $490(2.5)$ & $102(1.8)$ & $138(2.6)$ & $90(1.7)$ & $820(2.3)$ \\
\hline \multicolumn{6}{|l|}{ Calendar year at index date, $n(\%)$} \\
\hline 1996-1999 & $8533(44.1)$ & $3120(53.6)$ & $2648(49.5)$ & $2197(41.0)$ & $16,498(46.0)$ \\
\hline $2000-2004$ & $3256(16.8)$ & $866(14.9)$ & $803(15.0)$ & $1066(19.9)$ & $5991(16.7)$ \\
\hline $2005-2009$ & $3161(16.3)$ & $724(12.4)$ & $802(15.0)$ & $873(16.3)$ & $5560(15.5)$ \\
\hline 2010-2014 & $2942(15.2)$ & $767(13.2)$ & $765(14.3)$ & $772(14.4)$ & $5246(14.6)$ \\
\hline $2015-2017 / 2018$ & $1468(7.6)$ & $348(6.0)$ & $334(6.2)$ & $449(8.4)$ & $2599(7.2)$ \\
\hline Person-years of follow-up - total & 225,877 & 75,834 & 68,035 & 61,552 & 431,299 \\
\hline \multicolumn{6}{|c|}{ Person-years of follow-up during periods of exposure to } \\
\hline Any DMDs & 24,970 & 11,890 & 14,191 & 12,239 & 63,290 \\
\hline Any first-generation DMDs & 21,023 & 11,170 & 11,691 & 10,721 & 54,605 \\
\hline Beta-interferon $^{c}$ & 15,326 & 8044 & 7702 & 5440 & 36,512 \\
\hline Glatiramer acetate & 5697 & 3125 & 3989 & 5281 & 18,092 \\
\hline Any second-generation DMDs & 3947 & 720 & 2500 & 1517 & 8685 \\
\hline Natalizumab & 831 & 143 & 641 & 133 & 1748 \\
\hline Fingolimod & 987 & 123 & 540 & 77 & 1726 \\
\hline Dimethyl fumarate & 1246 & 326 & 795 & 980 & 3348 \\
\hline Teriflunomide & 657 & 125 & 485 & 270 & 1538 \\
\hline Alemtuzumab & 223 & 3 & 34 & 58 & 317 \\
\hline Daclizumab & $<6$ & 0 & 0 & 0 & $<6$ \\
\hline Ocrelizumab & $<6$ & 0 & $<6$ & 0 & $<6$ \\
\hline No DMD & 200,907 & 63,945 & 53,844 & 49,313 & 368,009 \\
\hline \multicolumn{6}{|c|}{ Number of individuals ever exposed, by type of DMD, during follow-up ${ }^{\mathrm{d}}, n(\%)$} \\
\hline Any DMDs & $4732(24.4)$ & $1762(30.2)$ & $2036(38.0)$ & $1888(35.2)$ & $10,418(29.0)$ \\
\hline First-generation DMDs -anye & $4124(21.3)$ & $1694(29.1)$ & $1763(32.9)$ & $1623(30.3)$ & $9204(25.6)$ \\
\hline Beta-interferon $^{c}$ & $3140(16.2)$ & $1294(22.2)$ & $1300(24.3)$ & $1019(19.0)$ & $6753(18.8)$ \\
\hline Glatiramer acetate & $1719(8.9)$ & $782(13.4)$ & $778(14.5)$ & $970(18.1)$ & $4249(11.8)$ \\
\hline Second-generation DMDs-any ${ }^{\mathrm{e}}$ & $1756(9.1)$ & $340(5.8)$ & $870(16.3)$ & $702(13.1)$ & $3668(10.2)$ \\
\hline Natalizumab & $286(1.5)$ & $52(0.9)$ & $207(3.9)$ & $49(0.9)$ & $594(1.7)$ \\
\hline Fingolimod & $421(2.2)$ & $69(1.2)$ & $201(3.8)$ & $42(0.8)$ & $733(2.0)$ \\
\hline Dimethyl fumarate & $758(3.9)$ & $193(3.3)$ & $360(6.7)$ & $518(9.7)$ & $1829(5.1)$ \\
\hline Teriflunomide & $520(2.7)$ & $86(1.5)$ & $260(4.9)$ & $194(3.6)$ & $1060(3.0)$ \\
\hline
\end{tabular}


Table 1. (Continued)

\begin{tabular}{|c|c|c|c|c|c|}
\hline Characteristics & $\begin{array}{l}\text { British } \\
\text { Columbia, } \\
n=19,360\end{array}$ & $\begin{array}{l}\text { Manitoba, } \\
n=5825\end{array}$ & $\begin{array}{l}\text { Nova Scotia, } \\
n=5352\end{array}$ & $\begin{array}{l}\text { Saskatchewan, } \\
n=5357\end{array}$ & $\begin{array}{l}\text { Overall cohort, } \\
n=35,894\end{array}$ \\
\hline Alemtuzumab & $179(0.9)$ & $6(0.1)$ & $39(0.7)$ & $71(1.3)$ & $295(0.8)$ \\
\hline Daclizumab & $6(<0.1)$ & 0 & 0 & 0 & $6(<0.1)$ \\
\hline Ocrelizumab & $<6(<0.1)$ & 0 & $7(0.1)$ & 0 & $7(<0.1)^{\mathrm{f}}$ \\
\hline \multicolumn{6}{|c|}{ 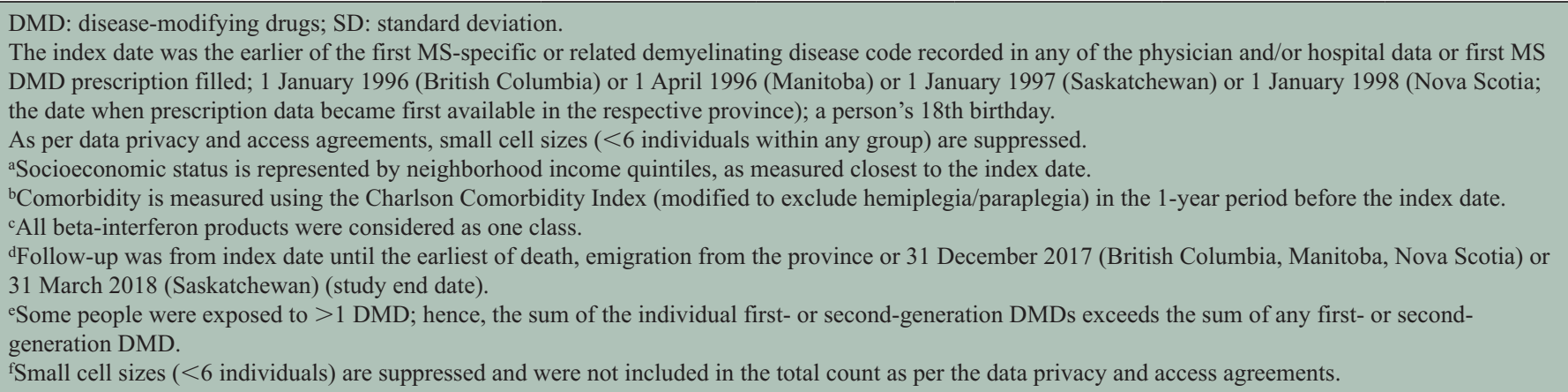 } \\
\hline
\end{tabular}

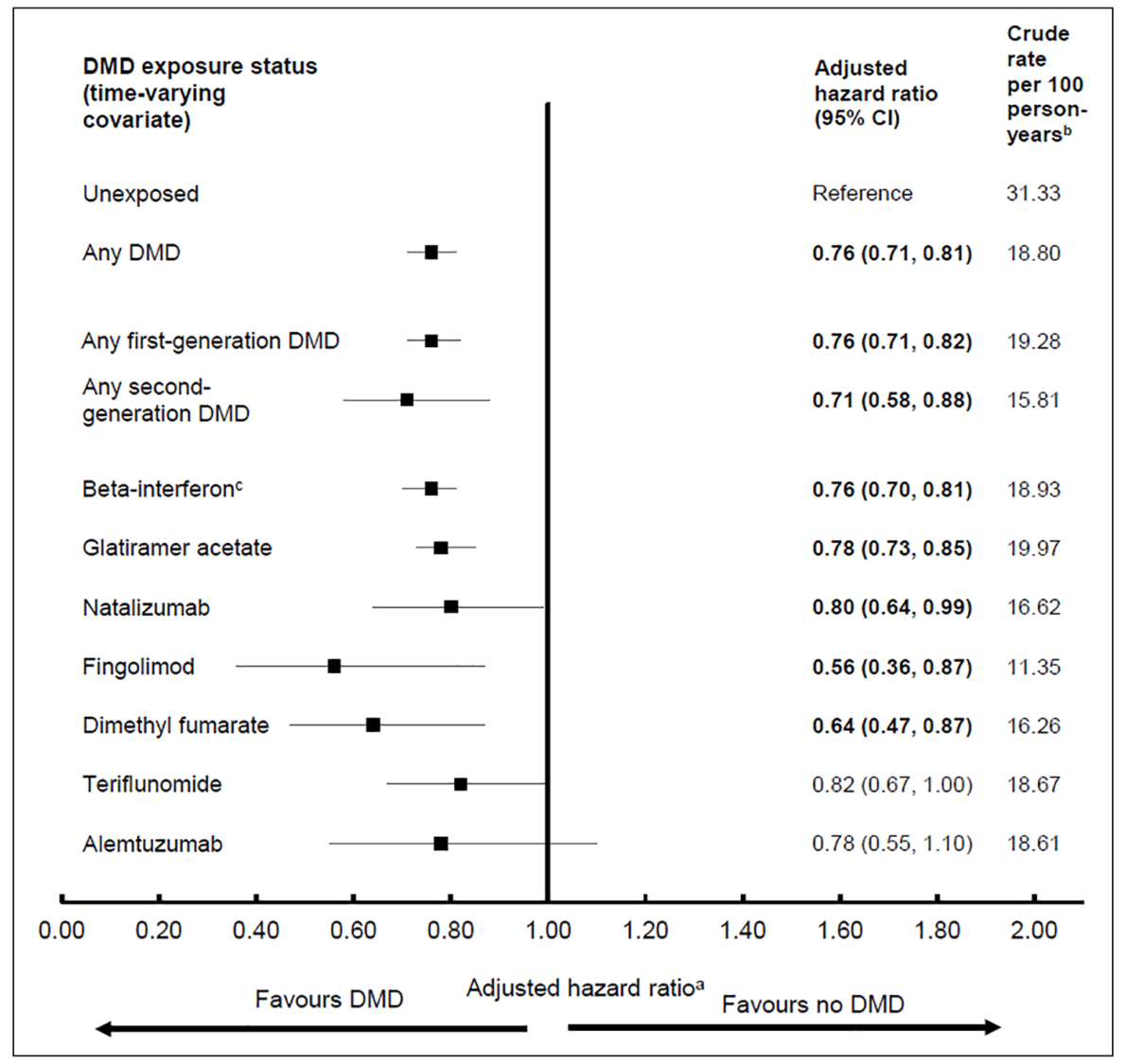

Figure 1. Exposure to disease-modifying drugs for multiple sclerosis and hazard of hospitalization in a population-based cohort.

CI: confidence interval; DMD: disease-modifying drug. Bold indicates $p<0.05$.

aResults from each of the four provinces were adjusted for sex, socioeconomic status (quintiles) closest to the index date, and the following characteristics over time: age (continuous), calendar year (continuous), and comorbidity score (categorized as $0,1,2, \geqslant 3$ ) measured using a modified Charlson Comorbidity Index and were then combined using random-effects meta-analyses.

bPerson-years of follow-up for the calculation of crude rate were as per Table 1 except the duration of a hospitalization was discounted from the follow-up time to avoid immortal time bias.

cAll beta-interferon products were considered as one class. 


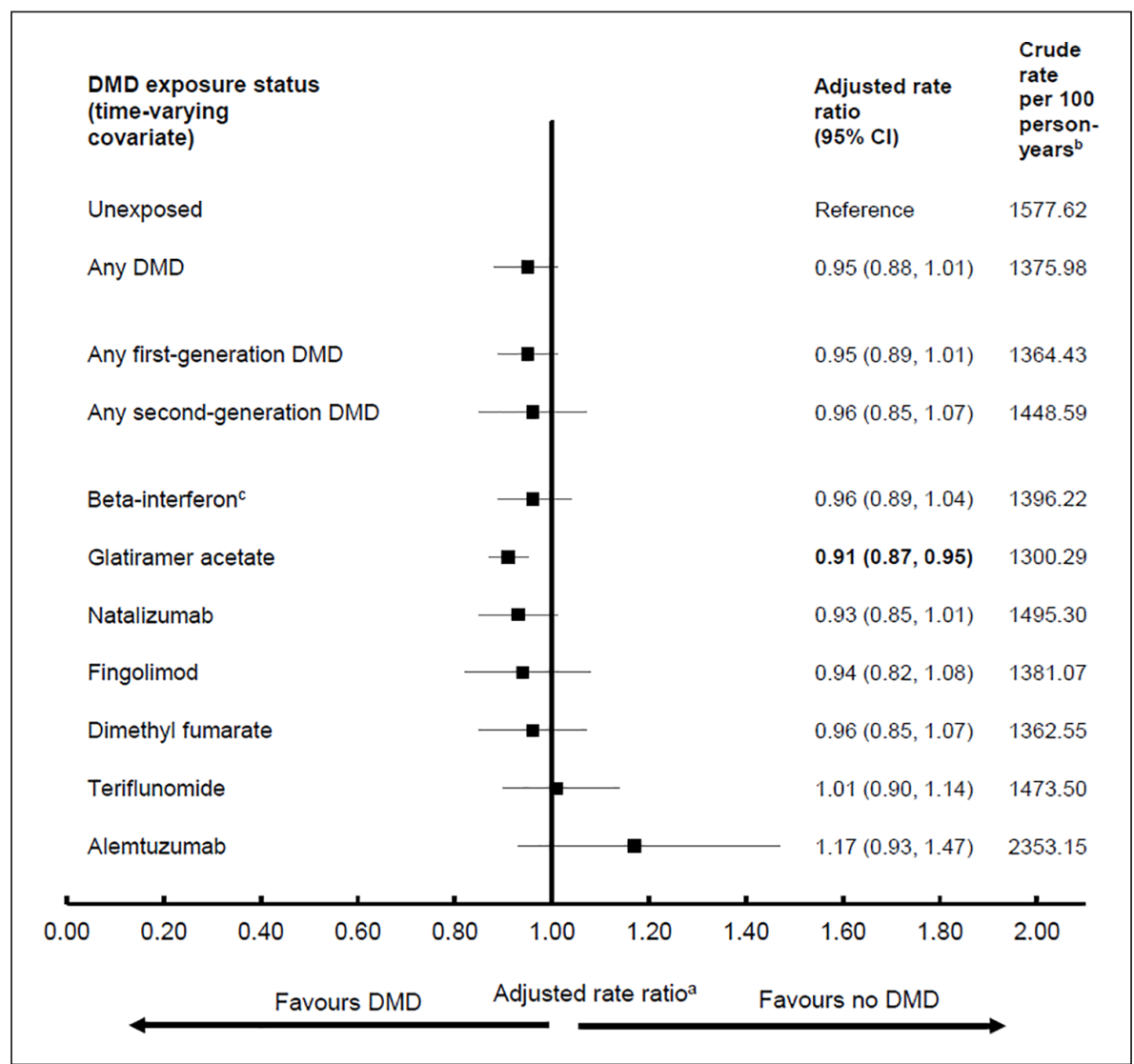

Figure 2. Exposure to disease-modifying drugs for multiple sclerosis and rates of physician service use. CI: confidence interval; DMD: disease-modifying drug. Bold indicates $p<0.05$.

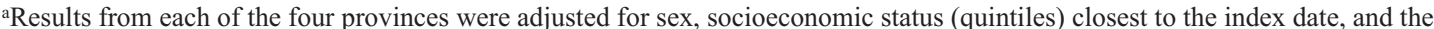
following characteristics over time: age (continuous), calendar year (continuous), and comorbidity score (categorized as $0,1,2, \geqslant 3$ ) measured using a modified Charlson Comorbidity Index and were then combined using random-effects meta-analyses. bPerson-years of follow-up/exposure are shown in Table 1 and were used to calculate the crude rates.

cAll beta-interferon products were considered as one class.

\section{Complementary analyses}

The direction of findings was generally similar for both sexes, although some estimates were more pronounced (lower) for males (Figures 3 and 4). For hospitalizations, this was most evident for the second-generation DMDs; in particular natalizumab was associated with a lower hazard for males (aHR: 0.62 ; 95\% CI: 0.47-0.81), but not for females (aHR: 0.93; 95\% CI: 0.64-1.35, Figure 3).

Crude rates of physician visits varied by individual DMD and relative to periods of no exposure (Figure 5). Fingolimod was associated with relatively high rates of ophthalmologist visits (57.17/100 person-years), while alemtuzumab with general practitioner (893.94/100 person-years), internal medicine (113.44/100 personyears), psychiatry (46.63/100 person-years), and physiatry visits (34.02/100 person-years). Periods of no exposure were associated with relatively high rates of general practitioner (949.70/100 person-years) and internal medicine visits (79.54/100 person-years) and three surgical specialty visits (general, neuro-, and orthopedic). When we included neurologist specialty in the overall number of physician visits the findings were consistent with those from the main analysis (using data from British Columbia only; Supplementary Figure 1).

\section{Discussion}

We examined the relationship between DMD exposure and health service use in a large population of individuals with MS in a universal healthcare setting, spanning $>20$ years. Exposure to any first-generation DMD was associated with a $24 \%$ lower hazard of hospitalization compared to no exposure, rising to $29 \%$ for the second-generation DMDs. Variation was seen across the individual DMDs, but all findings were 
Table 2. Disease-modifying drugs for multiple sclerosis: exposure by DMD and hazard of hospitalization (pairwise comparisons).

\begin{tabular}{|c|c|c|c|c|c|c|c|}
\hline \multirow{3}{*}{$\begin{array}{l}\text { DMD exposure } \\
\text { status (time-varying } \\
\text { covariate) }\end{array}$} & \multicolumn{7}{|c|}{ Reference category } \\
\hline & $\begin{array}{l}\text { Beta- } \\
\text { interferon }\end{array}$ & $\begin{array}{l}\text { Glatiramer } \\
\text { acetate }\end{array}$ & Natalizumab & Fingolimod & $\begin{array}{l}\text { Dimethyl } \\
\text { fumarate }\end{array}$ & Teriflunomide & Alemtuzumab \\
\hline & \multicolumn{7}{|c|}{ Adjusted hazard ratio $(95 \% \mathrm{CI})^{\mathrm{a}}$} \\
\hline Beta-interferon ${ }^{b}$ & - & $\begin{array}{l}0.96 \\
(0.90-1.03)\end{array}$ & $\begin{array}{l}0.97 \\
(0.79-1.18)\end{array}$ & $\begin{array}{l}1.35 \\
(0.83-2.20)\end{array}$ & $\begin{array}{l}1.18 \\
(0.88-1.59)\end{array}$ & $\begin{array}{l}0.91 \\
(0.71-1.18)\end{array}$ & $\begin{array}{l}1.01 \\
(0.72-1.43)\end{array}$ \\
\hline Glatiramer acetate & $\begin{array}{l}1.04 \\
(0.97-1.12)\end{array}$ & - & $\begin{array}{l}1.02 \\
(0.86-1.21)\end{array}$ & $\begin{array}{l}1.41 \\
(0.89-2.23)\end{array}$ & $\begin{array}{l}1.23 \\
(0.90-1.66)\end{array}$ & $\begin{array}{l}0.95 \\
(0.72-1.24)\end{array}$ & $\begin{array}{l}1.07 \\
(0.76-1.52)\end{array}$ \\
\hline Natalizumab & $\begin{array}{l}1.03 \\
(0.85-1.26)\end{array}$ & $\begin{array}{l}0.98 \\
(0.82-1.16)\end{array}$ & - & $\begin{array}{l}1.49 \\
(1.13-1.94)\end{array}$ & $\begin{array}{l}1.30 \\
(1.05-1.60)\end{array}$ & $\begin{array}{l}1.08 \\
(0.85-1.37)\end{array}$ & $\begin{array}{l}1.14 \\
(0.74-1.74)\end{array}$ \\
\hline Fingolimod & $\begin{array}{l}0.74 \\
(0.45-1.20)\end{array}$ & $\begin{array}{l}0.71 \\
(0.45-1.12)\end{array}$ & $\begin{array}{l}0.67 \\
(0.51-0.88)\end{array}$ & - & $\begin{array}{l}0.90 \\
(0.72-1.12)\end{array}$ & $\begin{array}{l}0.71 \\
(0.51-1.01)\end{array}$ & $\begin{array}{l}0.75 \\
(0.50-1.13)\end{array}$ \\
\hline Dimethyl fumarate & $\begin{array}{l}0.85 \\
(0.63-1.14)\end{array}$ & $\begin{array}{l}0.82 \\
(0.60-1.11)\end{array}$ & $\begin{array}{l}0.77 \\
(0.62-0.95)\end{array}$ & $\begin{array}{l}1.11 \\
(0.89-1.39)\end{array}$ & - & $\begin{array}{l}0.80 \\
(0.59-1.08)\end{array}$ & $\begin{array}{l}0.96 \\
(0.62-1.47)\end{array}$ \\
\hline Teriflunomide & $\begin{array}{l}1.09 \\
(0.85-1.42)\end{array}$ & $\begin{array}{l}1.05 \\
(0.80-1.38)\end{array}$ & $\begin{array}{l}0.93 \\
(0.73-1.18)\end{array}$ & $\begin{array}{l}1.40 \\
(0.99-1.98)\end{array}$ & $\begin{array}{l}1.25 \\
(0.93-1.69)\end{array}$ & - & $\begin{array}{l}1.05 \\
(0.63-1.75)\end{array}$ \\
\hline Alemtuzumab & $\begin{array}{l}0.99 \\
(0.70-1.39)\end{array}$ & $\begin{array}{l}0.93 \\
(0.66-1.32)\end{array}$ & $\begin{array}{l}0.88 \\
(0.57-1.35)\end{array}$ & $\begin{array}{l}1.33 \\
(0.89-2.00)\end{array}$ & $\begin{array}{l}1.05 \\
(0.68-1.61)\end{array}$ & $\begin{array}{l}0.96 \\
(0.57-1.60)\end{array}$ & - \\
\hline
\end{tabular}

CI: confidence interval; DMD: disease-modifying drug. Bold indicates $p<0.05$.

aResults from each of the four provinces were adjusted for sex, socioeconomic status (quintiles) closest to the index date, and the following characteristics over time: age (continuous), calendar year (continuous), and comorbidity score measured using a modified Charlson Comorbidity Index (categorized as 0,1 , $2, \geqslant 3)$ and were then combined using random-effects meta-analyses.

${ }^{\mathrm{b}}$ All beta-interferon products were considered as one class.

Table 3. Disease-modifying drugs for multiple sclerosis: exposure by DMD and rates of physician service use (pairwise comparisons).

\begin{tabular}{|c|c|c|c|c|c|c|c|}
\hline \multirow{3}{*}{$\begin{array}{l}\text { DMD exposure } \\
\text { status (time- } \\
\text { varying covariate) }\end{array}$} & \multicolumn{7}{|c|}{ Reference category } \\
\hline & $\begin{array}{l}\text { Beta- } \\
\text { interferon }\end{array}$ & $\begin{array}{l}\text { Glatiramer } \\
\text { acetate }\end{array}$ & Natalizumab & Fingolimod & $\begin{array}{l}\text { Dimethyl } \\
\text { fumarate }\end{array}$ & Teriflunomide & Alemtuzumab \\
\hline & \multicolumn{7}{|c|}{ Adjusted rate ratio $(95 \% \mathrm{CI})^{\mathrm{a}}$} \\
\hline Beta-interferon $^{\mathrm{b}}$ & - & $\begin{array}{l}1.06 \\
(1.00-1.13)\end{array}$ & $\begin{array}{l}1.08 \\
(1.00-1.17)\end{array}$ & $\begin{array}{l}1.03 \\
(0.89-1.20)\end{array}$ & $\begin{array}{l}1.01 \\
(0.93-1.09)\end{array}$ & $\begin{array}{l}0.95 \\
(0.87-1.04)\end{array}$ & $\begin{array}{l}0.82 \\
(0.74-0.91)\end{array}$ \\
\hline Glatiramer acetate & $\begin{array}{l}0.94 \\
(0.89-1.00)\end{array}$ & - & $\begin{array}{l}0.98 \\
(0.91-1.06)\end{array}$ & $\begin{array}{l}0.97 \\
(0.87-1.08)\end{array}$ & $\begin{array}{l}0.95 \\
(0.87-1.04)\end{array}$ & $\begin{array}{l}0.90 \\
(0.83-0.96)\end{array}$ & $\begin{array}{l}0.77 \\
(0.64-0.94)\end{array}$ \\
\hline Natalizumab & $\begin{array}{l}0.92 \\
(0.86-1.00)\end{array}$ & $\begin{array}{l}1.02 \\
(0.95-1.10)\end{array}$ & - & $\begin{array}{l}0.99 \\
(0.88-1.12)\end{array}$ & $\begin{array}{l}0.95 \\
(0.88-1.03)\end{array}$ & $\begin{array}{l}0.91 \\
(0.83-0.99)\end{array}$ & $\begin{array}{l}0.75 \\
(0.66-0.85)\end{array}$ \\
\hline Fingolimod & $\begin{array}{l}0.97 \\
(0.83-1.12)\end{array}$ & $\begin{array}{l}1.03 \\
(0.93-1.15)\end{array}$ & $\begin{array}{l}1.01 \\
(0.90-1.14)\end{array}$ & - & $\begin{array}{l}0.95 \\
(0.88-1.02)\end{array}$ & $\begin{array}{l}0.90 \\
(0.84-0.97)\end{array}$ & $\begin{array}{l}0.77 \\
(\mathbf{0 . 6 4 - 0 . 9 2 )}\end{array}$ \\
\hline Dimethyl fumarate & $\begin{array}{l}0.99 \\
(0.92-1.07)\end{array}$ & $\begin{array}{l}1.05 \\
(0.96-1.15)\end{array}$ & $\begin{array}{l}1.05 \\
(0.97-1.14)\end{array}$ & $\begin{array}{l}1.06 \\
(0.98-1.14)\end{array}$ & - & $\begin{array}{l}0.95 \\
(0.90-1.00)\end{array}$ & $\begin{array}{l}0.80 \\
(0.70-0.91)\end{array}$ \\
\hline Teriflunomide & $\begin{array}{l}1.05 \\
(0.96-1.14)\end{array}$ & $\begin{array}{l}1.12 \\
(1.04-1.20)\end{array}$ & $\begin{array}{l}1.10 \\
(1.01-1.20)\end{array}$ & $\begin{array}{l}1.11 \\
(1.03-1.19)\end{array}$ & $\begin{array}{l}1.06 \\
(1.00-1.11)\end{array}$ & - & $\begin{array}{l}0.84 \\
(0.73-0.96)\end{array}$ \\
\hline Alemtuzumab & $\begin{array}{l}1.22 \\
(1.10-1.35)\end{array}$ & $\begin{array}{l}1.29 \\
(1.07-1.57)\end{array}$ & $\begin{array}{l}1.34 \\
(1.17-1.52)\end{array}$ & $\begin{array}{l}1.30 \\
(1.08-1.57)\end{array}$ & $\begin{array}{l}1.25 \\
(1.10-1.43)\end{array}$ & $\begin{array}{l}1.19 \\
(1.04-1.37)\end{array}$ & - \\
\hline \multicolumn{8}{|c|}{$\begin{array}{l}\text { CI: confidence interval; DMD: disease-modifying drug. Bold indicates } p<0.05 \text {. } \\
\text { aResults from each of the four provinces were adjusted for sex, socioeconomic status (quintiles) closest to the index date, and the following characteristics } \\
\text { over time: age (continuous), calendar year (continuous), and comorbidity score measured using a modified Charlson Comorbidity Index (categorized as } 0,1 \text {, } \\
2, \geqslant 3 \text { ), and were then combined using random-effects meta-analyses. } \\
\text { bAll beta-interferon products were considered as one class. }\end{array}$} \\
\hline
\end{tabular}




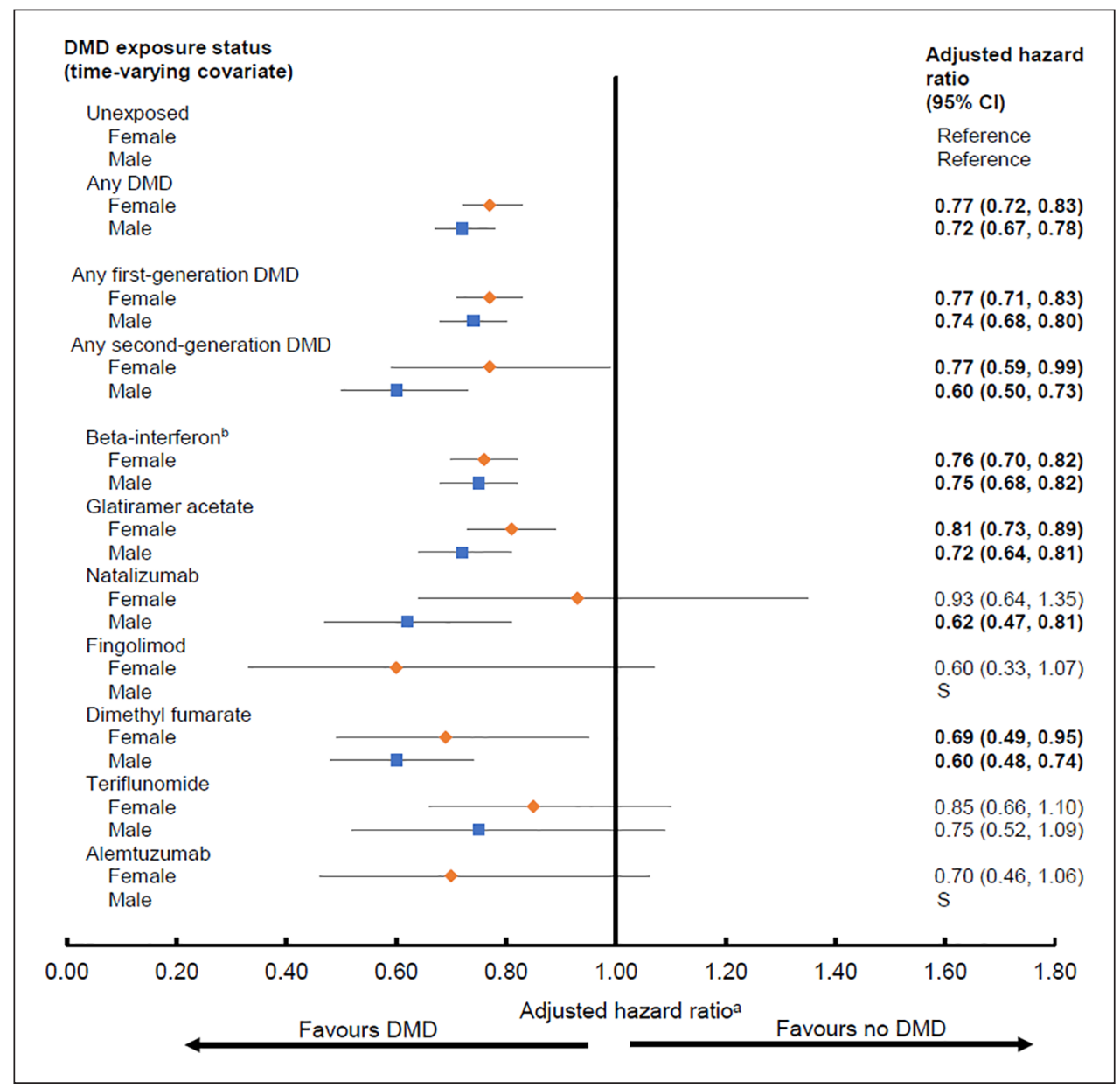

Figure 3. Exposure to disease-modifying drugs for multiple sclerosis and hazard of hospitalization by sex. CI: confidence interval; DMD: disease-modifying drug; S: small event rates prevented generation of reliable estimates. Bold indicates $p<0.05$.

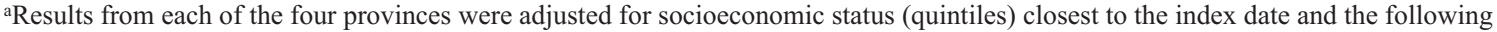
characteristics over time: age (continuous), calendar year (continuous), and comorbidity score (categorized as $0,1,2, \geqslant 3$ ) measured using a modified Charlson Comorbidity Index and were then combined using random-effects meta-analyses. Hazard ratios were estimated by introducing interaction terms between sex and DMD exposure variables.

${ }^{\mathrm{b}}$ All beta-interferon products were considered as one class.

suggestive of lower hospitalizations, ranging from $18 \%$ for teriflunomide to $44 \%$ for fingolimod. In contrast, DMD exposure (whether assessed by generation or individual DMD) versus no exposure was generally not associated with substantial differences in the overall rate of physician visits. Our study provides realworld evidence of a beneficial relationship between DMD exposure and hospitalizations.

The relationship between DMD exposure and healthcare use may be explained by several factors. The DMDs have been shown to reduce the frequency and severity of relapses, which in turn could lower the risk of hospitalization., ${ }^{4,521,22}$ Conversely, the DMDs are associated with various adverse events (AEs), including rare, but severe, events which may also pose a risk for hospitalization ${ }^{23}$ or may require regular physician visits for management and monitoring. ${ }^{24}$ We found relatively few studies with which to compare our findings. ${ }^{4-7,25}$ For example, two studies (from Saskatchewan, Canada, and Finland) took a broad ecological approach and examined the relationship between DMD and healthcare use at the population rather than individual level.,6,7 In Saskatchewan, as the overall number of filled DMD prescriptions increased all-cause hospitalizations decreased (1997-2016), while there was no relationship with the number of physician service claims. ${ }^{6}$ In Finland, a 4\% annual reduction in MS-related hospitalizations (2004-2014) coincided with the increased availability and utilization of the DMDs. ${ }^{7}$ Which individual DMDs were contributing to these patterns was not examined in 


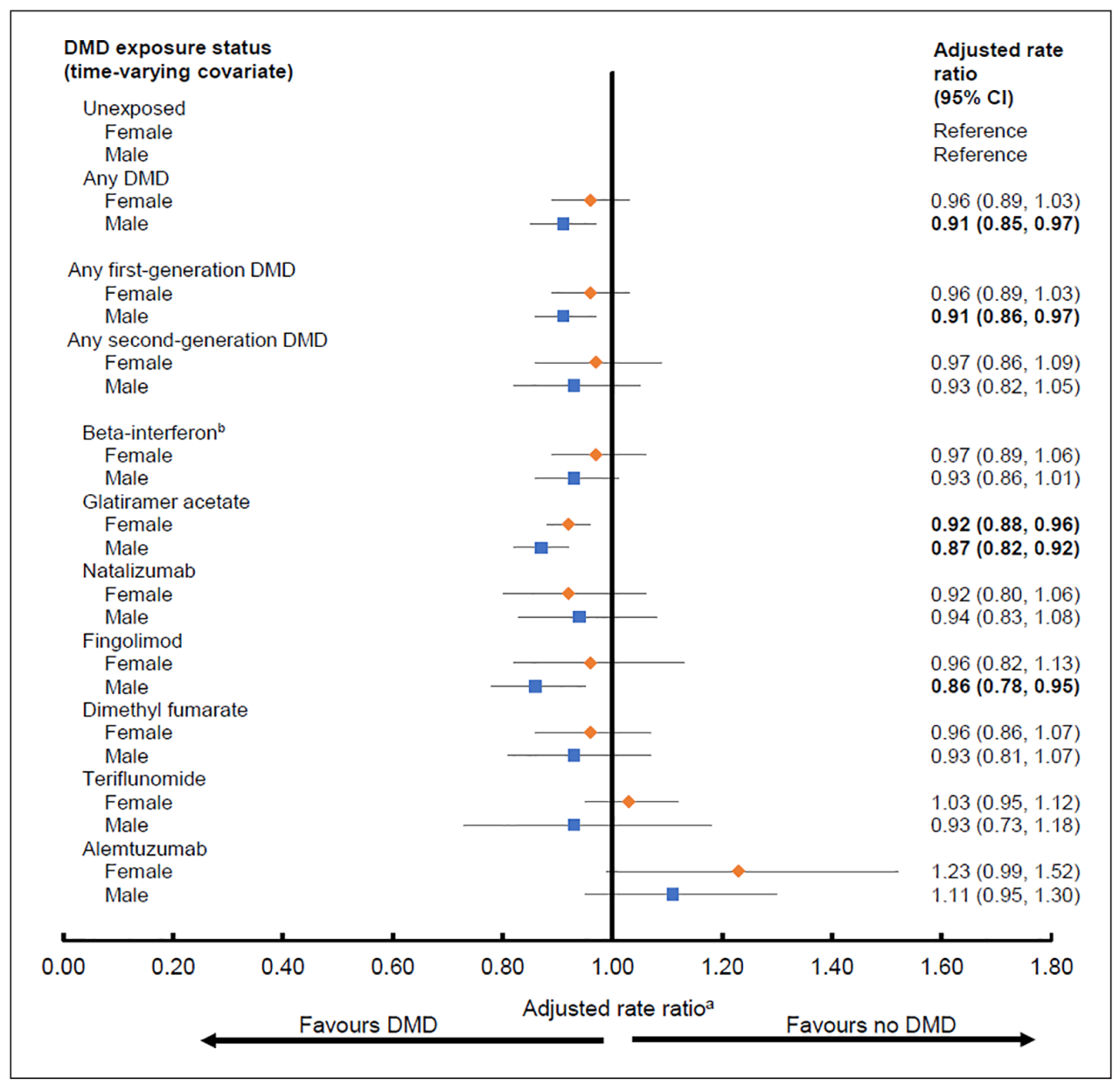

Figure 4. Exposure to disease-modifying drugs for multiple sclerosis and rates of physician service use by sex. CI, confidence interval; DMD, disease-modifying drug. Bold indicates $p<0.05$.

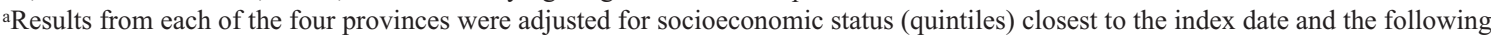
characteristics over time: age (continuous), calendar year (continuous), and comorbidity score (categorized as $0,1,2, \geqslant 3$ ) measured using a modified Charlson Comorbidity Index and were then combined using random-effects meta-analyses. Rate ratios were estimated by introducing interaction terms between sex and DMD exposure variables. ${ }^{b}$ All beta-interferon products were considered as one class.

either study. ${ }^{6,7}$ Two US studies (funded by the same pharmaceutical company) accessed data spanning a 2-year period on select groups of people with private insurance. ${ }^{4,5}$ They found a lower proportion of people hospitalized in the year after (versus before) DMD initiation. Neither study could assess whether participants left the insurance plan during follow-up and data beyond 2 years were unavailable. ${ }^{4,5} \mathrm{~A}$ third US-based study of Medicare enrollees reported fewer all-cause hospitalizations related to DMD use; however, interpretation of findings is limited due to its cross-sectional design and 1-year study period. ${ }^{25}$

Interesting differences emerged when the individual DMDs were directly compared to each other as an exploratory approach. For example, of the second-generation DMDs, natalizumab was associated with higher hazard of hospitalization versus two of the oral DMDs (fingolimod and dimethyl fumarate). Our findings might reflect natalizumab's status as a second-line treatment in Canada; it is typically reserved for patients with an inadequate response to another DMD due to its risk-benefit profile. ${ }^{2}$ This could mean that patients with more active disease might be exposed to natalizumab, which could result in more hospitalizations due to underlying disease activity. Some studies have compared natalizumab with fingolimod in relation to effectiveness, assessed by relapse rates and disability progression, but the findings have been mixed; few studies reported no differences, ${ }^{26,27}$ while others concluded that natalizumab seemed more effective than fingolimod, at least over the short term. ${ }^{28,29}$ Exposure to two DMDs - alemtuzumab and teriflunomide - was associated with higher rates of physician visits in our study. Rates were 19\%-34\% higher for alemtuzumab compared with each of the other DMDs, which likely 


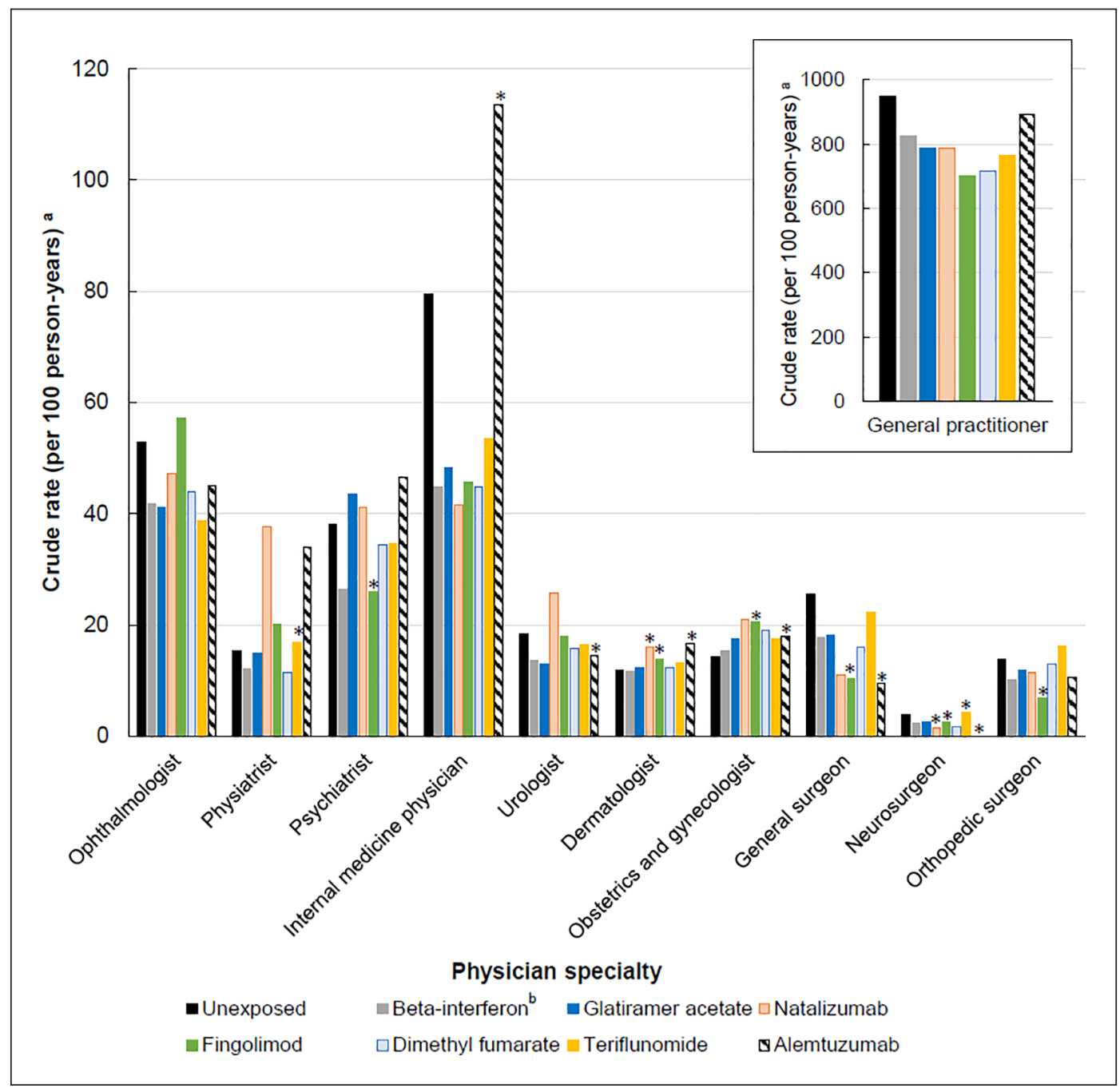

Figure 5. Exposure to disease-modifying drugs for multiple sclerosis and rates of physician visits by physician specialties.

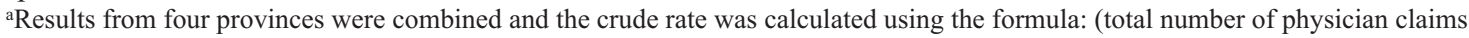
for each specialty by DMD exposure status/total person years of follow-up by DMD exposure status) $\times 100$. The results for general practitioner were shown in the right square box with a separate $y$-axis value (i.e. with a 10 times higher $y$-axis value than the remaining physician specialties).

${ }^{\mathrm{b}}$ All beta-interferon products were considered as one class.

*As per the data privacy and access agreements, small cell size ( $<6$ total number of physician claims reported in one or more provinces) is suppressed and were not included in the total count for that estimation of crude rate.

reflects the intensive (monthly) monitoring and laboratory testing required. ${ }^{30}$ Whereas for teriflunomide, rates were $10 \%-12 \%$ higher relative to glatiramer acetate, natalizumab, and fingolimod. For example, teriflunomide requires frequent blood tests, especially during the first 6 months of treatment, ${ }^{31}$ while glatiramer acetate does not. ${ }^{24}$ Frequent laboratory testing can also result in incidental findings, unrelated to $\mathrm{DMD}$, which in turn may necessitate further investigations and physician visits. ${ }^{32}$ Teriflunomide has been shown as less efficacious ${ }^{1}$ and less effective ${ }^{33}$ than either fingolimod or natalizumab in managing disease activity, which might also partly contribute to our observations. Thus, it is plausible that any increases in safety monitoring may be counterbalanced by a decrease in the need for physician services related to drug (in)effectiveness.

We also examined crude rates of physician services by specialty. Exposure to fingolimod was associated with relatively high rates of ophthalmologist visits, which would concur with the recommendation for regular ocular assessments and risk of macular edema. ${ }^{34}$ Alemtuzumab exposure was associated with high rates 
of general practitioner and internal medicine visits, likely reflecting the regular safety-related monitoring required and its $\mathrm{AE}$ profile, including risk of autoimmune disease. ${ }^{30}$ Why alemtuzumab was also associated with high rates of psychiatric visits was less clear. A systematic review found no significant association between any individual DMD, including alemtuzumab (based on randomized-controlled trials), and an increased risk of adverse psychiatric effects. ${ }^{35}$

Although our findings were generally similar for both males and females, there was some suggestion that the effects were more pronounced for males. We were unable to find another study with which to compare these sex-specific findings. DMD-related sex differences appears poorly understood, despite well-recognized sexdifferences in pharmacokinetics/pharmacodynamics. ${ }^{36,37}$ A 2018 review found that the majority $(65 \%)$ of MS clinical trials did not pre-plan a sex-specific analysis. ${ }^{37}$ Of those that did, only efficacy was examined (not harms) and while most found no sex differences, studies may have lacked sufficient statistical power as relatively few males were included. ${ }^{36,37}$ Although males comprised approximately $30 \%$ of individuals in our study, our cohort was sizable. Nonetheless, our cohort was insufficient to reliably examine sex differences for the more recent DMDs.

Our study has several limitations. While administrative health data offer opportunity to examine the patterns of health service utilization in the MS population at the individual level, these patterns should be interpreted accordingly; health services use may reflect, in part, a balance between the potential harms and benefits of the DMDs and should not be interpreted as only representing one or the other. We were not able to consider MS-specific clinical characteristics, such as relapses or disability which are not captured in the administrative data. Despite adjusting for several demographic characteristics and comorbidity, it is possible that comparisons between individual DMDs were influenced by underlying clinical features or other unmeasured confounders. For example, patients with more active disease might be treated with a higher efficacy DMD, but might also have required frequent physician visits or inpatient management due to their underlying disease. However, our complementary analyses, in which we included neurologist visits, did not change interpretation of findings. We had limited power to assess some of the newer second-generation DMDs which did not become widely available until close to our study end. Consequently, for DMDs such as alemtuzumab, the total personyears exposed was modest. We did not explore causespecific events as, particularly for hospitalizations, modest number of events would prevent generation of reliable estimates. Other information such as lifestyle behaviors (e.g. smoking) or race/ethnicity or access to DMD as part of a clinical trial were not available. For example, aside from a very small number of individuals that may have enrolled in a clinical trial and been randomized to receive a DMD, most individuals would not have been on a DMD before the index date. ${ }^{2}$ Nonetheless, we were able to account for several important factors which may influence treatment decisions and outcomes, such as socioeconomic status, sex, age, comorbidity burden, and calendar-year over time. We used a time-dependent approach when examining DMD exposure, which accounts for the changing treatment status of persons over time (an important consideration in minimizing bias, for example, immortal time bias). It could be value for future studies to explore different ways of grouping the DMDs (e.g. by relative efficacy or by mechanism of action). Other study strengths included avoidance of selection bias (by accessing objectively collected population-based data) and a long follow-up duration. The universal healthcare setting allowed us to provide a comprehensive evaluation of health service utilization, including all hospital admission, physician visits, and filled prescriptions, regardless of a person's ability to pay. Few world regions have access to such comprehensive, linked administrative health data for the entire population at the individual level.

\section{Conclusion}

We found in our study that periods of exposure to any DMD, or any first- or second-generation DMD, were associated with a lower hazard of hospitalization relative to no DMD exposure. However, there was substantial variation across the individual DMDs, particularly for the second-generation DMDs. In contrast, the relationship between DMD exposure with overall rates of physician visits was rather modest. Our study provides real-world insights into the beneficial effects of different DMDs on hospitalizations for people living with MS.

\section{Acknowledgements}

We are grateful to the Data Services Platform of the Saskatchewan Centre for Patient-Oriented Research (SCPOR). We are also grateful to Yan Wang (Dalhousie University) for her support in performing data analyses in Nova Scotia.

Access to, and use of, $\mathrm{BC}$ data was facilitated by Population Data BC and approved by the BC Ministry of Health, BC PharmaNet, and the BC Vital Statistics Agency. The authors acknowledge the Manitoba Centre for Health Policy for use of the Population 
Research Data Repository under project 2018-023 (HIPC 2018/19-13). Some data used in this report were made available by Health Data Nova Scotia of Dalhousie University. All inferences, opinions, and conclusions drawn in this manuscript are those of the authors and do not reflect the opinions or policies of the British Columbia Data Steward(s), Manitoba Centre for Health Policy or Manitoba Health, Health Data Nova Scotia, or the Nova Scotia Department of Health and Wellness. This study is based, in part, on de-identified data provided by the Saskatchewan Ministry of Health and eHealth Saskatchewan. The interpretation and conclusions contained herein do not necessarily represent those of the Government of Saskatchewan, the Saskatchewan Ministry of Health, or eHealth Saskatchewan.

\section{Data Availability Statement}

As we are not the data custodians, we are not authorized to make the data available. With the appropriate approvals, the data may be accessed through the Population Data British Columbia, Saskatchewan Health Quality Council, Manitoba Centre for Health Policy, and Health Data Nova Scotia of Dalhousie University.

\section{Declaration of Conflicting Interests}

The author(s) declared the following potential conflicts of interest with respect to the research, authorship, and/or publication of this article: H.S.N. receives funding from the Multiple Sclerosis Society of Canada's endMS Postdoctoral Fellowship and the Michael Smith Foundation for Health Research Trainee Award. During the past year, H.S.N. has received funding from the Canadian Institutes of Health Research (CIHR) Drug Safety and Effectiveness Cross-Disciplinary Training Program. F.Z. has no conflicts of interests relevant to this study. E.K. has no conflicts of interests relevant to this study. Y.Z. has no conflicts of interests relevant to this study. S.Y. has no conflicts of interests relevant to this study. O.E. has no conflicts of interests relevant to this study. L.W.S. has no conflicts of interests relevant to this study. C.E. receives funding from CIHR. J.D.F. receives research funding from CIHR, Multiple Sclerosis Society of Canada, Crohn's and Colitis Canada, Research Nova Scotia; consultation and distribution royalties from MAPI Research Trust. R.A.M. receives research funding from CIHR, Research Manitoba, Multiple Sclerosis Society of Canada, Multiple Sclerosis Scientific Foundation, Crohn's and Colitis Canada, National Multiple Sclerosis Society, CMSC and the US Department of Defense, and is a co-investigator on studies receiving funding from Biogen Idec and Roche Canada. H.T. is the Canada Research Chair for Neuroepidemiology and Multiple Sclerosis. Current research support received from the National Multiple Sclerosis Society, the Canadian Institutes of Health Research, the Multiple Sclerosis Society of Canada, and the Multiple Sclerosis Scientific Research Foundation. In addition, in the last 5 years, has received research support from the UK MS Trust; travel expenses to present at CME conferences from the Consortium of MS Centres (2018), the National MS Society (2016, 2018), ECTRIMS/ACTRIMS (2015, 2016, 2017, 2018, 2019, 2020), and American Academy of Neurology (2015, 2016, 2019). Speaker honoraria are either declined or donated to an MS charity or to an unrestricted grant for use by H.T.'s research group.

\section{Funding}

The author(s) disclosed receipt of the following financial support for the research, authorship, and/or publication of this article: This study was supported by the Canadian Institutes of Health Research (CIHR) Project and Foundation grant (PJT-156363 and FDN159934, PI: Tremlett).

\section{ORCID iDs}

Huah Shin Ng (DD https://orcid.org/0000-0001-83815253

Ruth Ann Marrie (iD https://orcid.org/0000-0002-18555595

Helen Tremlett (iD https://orcid.org/0000-0001-58042535

\section{Study Registration and Ethics Standards}

This study was registered with ClinicalTrials.gov (NCT04472975) and approvals were obtained from the Research Ethics Boards at the University of British Columbia and University of Saskatchewan (harmonized ethics: H18-00407), University of Manitoba (HS21764), and Nova Scotia Health Authority (1023555).

\section{Supplemental Material}

Supplemental material for this article is available online.

\section{References}

1. Tramacere I, Del Giovane C, Salanti G, et al. Immunomodulators and immunosuppressants for relapsing-remitting multiple sclerosis: A network meta-analysis. Cochrane Datab Syst Rev 2015; 2015: CD14011381.

2. Ng HS, Zhu F, Kingwell E, et al. Characteristics of a population-based multiple sclerosis cohort 
treated with disease-modifying drugs in a universal healthcare setting. Expert Rev Neurother 2020; 21: 131-140.

3. Marrie RA, Elliott L, Marriott J, et al. Dramatically changing rates and reasons for hospitalization in multiple sclerosis. Neurology 2014; 83: 929-937.

4. Nicholas J, Boster A, Wu N, et al. Comparison of disease-modifying therapies for the management of multiple sclerosis: Analysis of healthcare resource utilization and relapse rates from US insurance claims data. Pharmacoecon Open 2018; 2(1): 31-41.

5. Bonafede MM, Johnson BH and Watson C. Health care-resource utilization before and after natalizumab initiation in multiple sclerosis patients in the US. Clinicoecon Outcomes Res 2013; 6: 11-20.

6. Al-Sakran L, Marrie RA, Blackburn D, et al. Association between disease-modifying therapies for multiple sclerosis and healthcare utilisation on a population level: A retrospective cohort study. BMJ Open 2019; 9: e033599.

7. Pirttisalo AL, Sipilä JOT, Soilu-Hänninen M, et al. Adult hospital admissions associated with multiple sclerosis in Finland in 2004-2014. Ann Med 2018; 50(4): 354-360.

8. Statistics Canada. Census profile, 2016 census, 2017, https://www12.statcan.gc.ca/censusrecensement/2016/dp-pd/prof/index.cfm?Lang=E (accessed 1 February 2021).

9. British Columbia Ministry of Health [Creator]. Medical services plan (MSP) payment information file: V2 - Population data BC [Publisher]. Data Extract. MOH, 2017, http://www.popdata.bc.ca/data

10. Canadian Institute for Health Information [Creator]. Discharge abstract database (hospital separations): V2-Population data BC [Publisher]. Data extract. MOH, 2017, http://www.popdata.bc.ca/data

11. British Columbia Ministry of Health [Creator]. Consolidation file (MSP registration \& premium billing): V2. Population Data BC [Publisher]. Data extract. MOH, 2017, http://www.popdata.bc.ca/data

12. BC and Vital Statistics Agency [Creator]. Vital statistics deaths: V2. Population data BC [Publisher]. Data extract. BC Vital Statistics Agency, 2017, http:// www.popdata.bc.ca/data

13. BC and Ministry of Health [Creator]. PharmaNet: V2. BC Ministry of Health [Publisher]. Data Extract. Data Stewardship Committee, 2017, http://www.popdata. bc.ca/data

14. Marrie RA, Yu N, Blanchard J, et al. The rising prevalence and changing age distribution of multiple sclerosis in Manitoba. Neurology 2010; 74: 465-471.

15. Canadian Institute for Health Information Statistics Canada. Health Indicators 2013, 2013, https://www. cihi.ca/sites/default/files/document/health-indicators2013-en.pdf (accessed 3 March 2021).

16. Marrie RA, Bernstein CN, Peschken CA, et al. Intensive care unit admission in multiple sclerosis: Increased incidence and increased mortality. Neurology 2014; 82: 2112-2119.

17. Wijnands JMA, Zhu F, Kingwell E, et al. Diseasemodifying drugs for multiple sclerosis and infection risk: A cohort study. J Neurol Neurosurg Psychiatry 2018; 89(10): 1050-1056.

18. Graham DJ, Staffa JA, Shatin D, et al. Incidence of hospitalized rhabdomyolysis in patients treated with lipid-lowering drugs. JAMA 2004; 292: 2585-2590.

19. Evans C, Zhu F, Kingwell E, et al. Association between beta-interferon exposure and hospital events in multiple sclerosis. Pharmacoepidemiol Drug Saf 2014; 23(11): 1213-1222.

20. Rae-Grant A, Day GS, Marrie RA, et al. Practice guideline recommendations summary: diseasemodifying therapies for adults with multiple sclerosis - Report of the guideline development, dissemination, and implementation Subcommittee of the American Academy of Neurology. Neurology 2018; 90: 777-788.

21. O'Connor PW, Lublin FD, Wolinsky JS, et al. Teriflunomide reduces relapse-related neurological sequelae, hospitalizations and steroid use. J Neurol 2013; 260(10): 2472-2480.

22. Weinstock-Guttman B, Galetta SL, Giovannoni G, et al. Additional efficacy endpoints from pivotal natalizumab trials in relapsing-remitting MS. J Neurol 2012; 259(5): 898-905.

23. Simbrich A, Thibaut J, Khil L, et al. Drug-use patterns and severe adverse events with diseasemodifying drugs in patients with multiple sclerosis: A cohort study based on German claims data. Neuropsychiatr Dis Treat 2019; 15: 1439-1457.

24. Rommer PS, Zettl UK, Kieseier B, et al. Requirement for safety monitoring for approved multiple sclerosis therapies: An overview. Clin Exp Immunol 2014; 175(3): 397-407.

25. Sanchirico M, Caldwell-Tarr A, Mudumby P, et al. Treatment patterns, healthcare resource utilization, and costs among Medicare patients with multiple sclerosis in relation to disease-modifying therapy and corticosteroid treatment. Neurol Ther 2019; 8(1): 121-133.

26. Koch-Henriksen N, Magyari M, Sellebjerg F, et al. A comparison of multiple sclerosis clinical disease activity between patients treated with natalizumab and fingolimod. Mult Scler 2017; 23(2): 234-241.

27. Lanzillo R, Carotenuto A, Moccia M, et al. A longitudinal real-life comparison study of 
Visit SAGE journals online journals.sagepub.com/ home $/ \mathrm{msj}$

(S) SAGE journals natalizumab and fingolimod. Acta Neurol Scand 2017; 136(3): 217-222.

28. Lorscheider J, Benkert P, Lienert C, et al. Comparative analysis of natalizumab versus fingolimod as secondline treatment in relapsing-remitting multiple sclerosis. Mult Scler 2018; 24(6): 777-785.

29. Kalincik T, Horakova D, Spelman T, et al. Switch to natalizumab versus fingolimod in active relapsingremitting multiple sclerosis. Ann Neurol 2015; 77(3): 425-435.

30. Sanofi Genzyme A and Division of SanofiAventis Canada Inc. LEMTRADA (Alemtuzumab $12 \mathrm{mg} / 1.2 \mathrm{~mL}$ ) product monograph [online], 2020, https://www.canada.ca/en/health-canada/services/ drugs-health-products/drug-products/drug-productdatabase.html (accessed 11 January 2021).

31. Sanofi Genzyme A and Division of Sanofi-Aventis Canada Inc. AUBAGIO (Teriflunomide tablets 14 $\mathrm{mg}$ ) product monograph [online], 2020, https://www. canada.ca/en/health-canada/services/drugs-healthproducts/drug-products/drug-product-database.html (accessed 11 January 2021).
32. Wians FH. Clinical laboratory tests: Which, why, and what do the results mean? Lab Med 2009; 40: 105-113.

33. Kalincik T, Kubala Havrdova E, Horakova D, et al. Comparison of fingolimod, dimethyl fumarate and teriflunomide for multiple sclerosis. J Neurol Neurosurg Psychiatry 2019; 90(4): 458-468.

34. Jain N and Bhatti MT. Fingolimod-associated macular edema: Incidence, detection, and management. Neurology 2012; 78: 672-680.

35. Gasim M, Bernstein CN, Graff LA, et al. Adverse psychiatric effects of disease-modifying therapies in multiple sclerosis: A systematic review. Mult Scler Relat Disord 2018; 26: 124-156.

36. Li R, Sun X, Shu Y, et al. Sex differences in outcomes of disease-modifying treatments for multiple sclerosis: A systematic review. Mult Scler Relat Disord 2017; 12: 23-28.

37. Houtchens MK and Bove R. A case for gender-based approach to multiple sclerosis therapeutics. Front Neuroendocrinol 2018; 50: 123-134. 